\title{
Improving domiciliary robotic services by integrating the ASTRO Robot in an AmI Infrastructure
}

\author{
Filippo Cavallo, Michela Aquilano, Manuele Bonaccorsi, Raffaele Limosani, \\ Alessandro Manzi, Maria Chiara Carrozza and Paolo Dario \\ The BioRobotics Institute of Scuola Superiore Sant'Anna, Pisa, Italy, \\ Corresponding Author: f.cavallo@sssup.it
}

\begin{abstract}
This work describes the ECHORD Experiment ASTROMOBILE, a project aimed to design, develop and test a system for favourable independent living, improved quality of life and efficiency of care for senior citizens in domestic environments. The system, composed of a mobile robotic platform (called ASTRO) and an Ambient Intelligent Infrastructure that actively cooperated between them and with the end-user, was designed and implemented with a user-centred design approach, involving different stakeholders. The system was designed to deliver services to users, like drug delivery, stand support, reminding, info-entertainment. The design took advantages of the integration of robotic platforms with smart environments, to provide to users higher quality and localization based services. Senior end-users were involved in the experimentation of the system in the DomoCasa Living Lab and feedbacks were gathered for the technology assessment. Particularly, this paper demonstrates the general feasibility of the ASTROMOBILE system and thanks to users feedbacks its acceptability and usability.
\end{abstract}

Keywords: Companion robot, ambient assisted living, autonomous robot, smart environment, ambient intelligence, user localization.

\section{Introduction}

Recent studies highlight that the global demographic trend of ageing people is rapidly growing. In 2007, it was underlined that the number of senior citizens was increasing by 8 million per year and by 2030 is expected to reach 24 million per year [1]. In order to provide assistance to senior citizens, the Society has to sustain high costs, that will increase in terms of medical cures, socio-medial services and social activities. In addition, the difficulty to manage this situation is also aggravated by the current financial crisis [2]. To induce important social and economical benefits, it is crucial to preserve the independence of the elderly, delay and reduce institutionalization and favour the participation to community life. Robotics and ICT technologies represent a profitable solution for more efficient management and delivery of health and social care, as well as improvement of 
independent living, quality of life and efficient cares and assist people to live longer and more comfortably in their own homes [3].

The idea of using robotic systems for domiciliary assistance has been investigated in different works that highlighted the potentiality to enhance the independent living of elderly people and provide health care assistance and support in their daily activities and to improve their well-being and efficiency in care through Ambient Assisted Living (AAL) services [4-10]. In such services, mobile robotic systems become a sort of companion (co-worker) that operates with end-users [11] and provides dedicated services anywhere and any time, thus requiring high interaction capabilities and dependability and acceptability features. As a matter of fact, a service based on mobile robotic systems requires some properties that allow to enhance the Quality of Service (QoS), among which:

1. technical efficacy in providing service with high robustness and safety;

2. efficiency and readiness in providing correct services to the users;

3. easy usability in using the service;

4. appropriate aesthetics and functionalities.

Recently some works demonstrated that the integration of mobile robotic systems in smart domestic environments could reduce the necessity of robots with a large set of complex on-board sensors [26] and consequently provide high quality, cost effective and safety services. One of the most important requirements for robotic systems is the ability to reason about the places and times when and where user is in the domestic environment in order to navigate toward him/her when the service is activated and the user is not in the proximity of robot, i.e. in another room. This task could be very difficult for a single robot because requires several complex ability and sensors to seek the user in the home; however a sensor network based on simple and affordable sensors can more appropriately detect the user and share the relative position with the robot that, as a consequence, can in real time know the position of the user and provide the service immediately and in a reasonable time.

The capability of a robot to efficiently seek the user by exploiting an Ambient Intelligent (AmI) infrastructure is still a present scientific challenge. In [12], a robot combined its laser range finder for legs recognition with environmental Presence Infrared Sensors (PIR) able to estimate the position of the user. In [13], a companion robot and a domestic sensor network was simulated to evaluate the advantages of using an ubiquitous robotic framework approach with respect to the traditional robot-centred approach. It was demonstrated through extensive tests that the companion robot, integrated in a sensor network for real-time user localization and home status monitoring, resulted feasible, efficient and time effective.

In this context, this work presents the ASTROMOBILE Experiment, which aimed to design, develop and test the ASTROMOBILE System for favourable independent living, improved quality of life and efficiency of care and to demonstrate its general feasibility, scientific and technological effectiveness and acceptability by end-users. Particularly this paper focuses on the description of 
the ASTROMOBILE system, composed of the ASTRO robot and an AmI Infrastructure, that used a top-down technique to localize user inside the domestic environment. Starting from a User Centered Design approach and after the analysis of the user requirements, specific design activities on the appearance and functionalities of the robotic platform were carried out. The use of an advanced human robot interface, based on natural language, enhanced the interaction and the usability of the ASTRO robot. In addition, the ability to exploit the information coming from the smart sensor network improved the behaviour of the robot in terms of quality and performance of the services.

Section 2 describes the User Centered Design approach. Section 3 shows the components of the ASTROMOBILE system and Section 4 highlights the deep interaction between the robot and smart environment. At the end, Section 5 gives the details of the experimental phase.

\section{Methodology}

The development of the ASTROMOBILE system and its relative services were performed using a User Centered Design approach in order to address the criteria of acceptability and usability, that have been demonstrated to be crucial for plausible deployment and exploitation in real socio-medical contexts[14]. For this purpose, 21 senior citizens of the Municipality of Peccioli, an Italian small town where the ASTROMOBILE Experiment was tested, were recruited and involved in the design, development and experimental process of the system. Particularly, the development was scheduled in the following phases (summarized in Fig. 1):

1. identification of end-users' needs and definition of the ASTROMOBILE system's requirements, functionalities and relative application scenarios (11 elderly);

2. identification of technological aspects for the prototyping of the ASTROMOBILE system;

3. development of ASTROMOBILE prototype (21 elderly);

4. experimentation and evaluation of the ASTROMOBILE system with senior citizens in the DomoCasa Living Lab (15 elderly).

The key point of this approach was the continuous involvement of elderly in almost all phases to make this research really effective in terms of acceptability and dependability. During the first phase, a focus group with 11 elderly was organized to create the first concept of the system; multiple- choice and free- response questionnaires were used to collect ideas and motivations on the functionalities of the ASTROMOBILE system (Table 1), on the different modalities to interact with it (natural language, touch screen, colored lights, remote controller) and on the appearance characteristics (shape, dimensions, colors and materials). During the second phase, the technicians identified the technological opportunities and challenges to implement such ASTROMOBILE system according to the endusers' requests and requirements. During the third phase, the ASTROMOBILE system was completely developed and, interestingly, 21 elderly participated in 


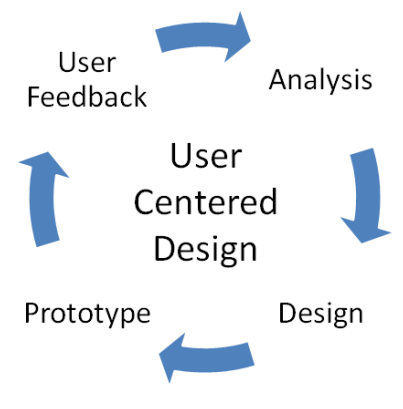

Fig. 1. Main developmental phases of ASTROMOBILE with an User Centred Design Approach.

training the speech recognition software on the most common 145 words of the scenarios. Finally 15 elderly were asked to concretely test the ASTROMOBILE system in the seven realistic scenarios of the DomoCasa Living Laboratory and answer to evaluation questionnaires.

\section{Description of the System}

The ASTROMOBILE system was composed of two main coexisting agents that were able to cooperate between them and with end-users:

- the ASTRO robot;

- an Ambient Intelligent ( $A m I)$ Infrastructure, implemented by means of two Wireless Sensor Networks (WSN), one for environmental monitoring, called Sensor Network $(S N)$, and another for localization, called Localization Network $(L N)$.

\subsection{ASTROMOBILE Architecture}

The ASTROMOBILE System was conceptually implemented as a modular architecture structured in four different layers (see Fig. 2):

- the physical layer, composed of the hardware components, such as the mobile platform, Wireless Sensor Networks, touch screen, speaker and microphone, smartphone, Inertial Measurement Unit and PC;

- the low-level processing and communication layer, composed of six functional modules (Locator, Navigator, Sensor, GUI, Speech and Inertial);

- the high-level planning layer, composed of the Astrologic module, that was conceived to implement easy algorithms of context awareness and decision making and manage high-level tasks, such as "agenda and reminder", "web interface control" and "Skype-like applications"; 
Table 1. Scenarios used in ASTROMOBILE project

\begin{tabular}{|c|c|c|}
\hline Service & Id & Scenarios \\
\hline \multirow{2}{*}{\multicolumn{2}{|c|}{$\begin{array}{l}\text { User needs ASTRO to S2 } \\
\text { carry out some daily ac- } \\
\text { tivities }\end{array}$}} & - The user needs ASTRO because he/she needs a \\
\hline & & $\begin{array}{l}\text { physical help to stand up from the sofa or the bed } \\
\text { - The user calls ASTRO because he/she needs one } \\
\text { of objects set on the ASTRO's pocket (TV remote } \\
\text { controller glass of water, etc.); }\end{array}$ \\
\hline & $\mathrm{S} 3$ & $\begin{array}{l}\text { - The user calls ASTRO to call caregiver because } \\
\text { he/she feels sick (Skype from bed); }\end{array}$ \\
\hline & $\mathrm{S} 4$ & $\begin{array}{l}\text { - The user calls ASTRO because he/she needs to ac- } \\
\text { cess to entertainment tools (music, pictures, videos, } \\
\text { etc.); }\end{array}$ \\
\hline \multirow{2}{*}{$\begin{array}{l}\text { ASTRO } \\
\text { autonomously helps user } \\
\text { in appropriate situations }\end{array}$} & S5 & $\begin{array}{l}\text { - ASTRO moves to user for reminding him/her to } \\
\text { take drugs or appointments; }\end{array}$ \\
\hline & S6 & $\begin{array}{l}\text { - ASTRO moves to user because there is a critical } \\
\text { alert in the house (i.e. the door is open while he/she } \\
\text { is in bedroom); }\end{array}$ \\
\hline $\begin{array}{l}\text { Caregiver remotely } \\
\text { drives ASTRO to } \\
\text { assist the user }\end{array}$ & S7 & $\begin{array}{l}\text { - The caregiver is outside the home and connects } \\
\text { remotely to ASTRO asking it to move to the user } \\
\text { and activate the webcam to assess and support the } \\
\text { user }\end{array}$ \\
\hline
\end{tabular}

- the service layer, represented by the different services that the system was able to provide (see Table 1).

The six modules of the low-level processing and communication layer were assigned to a specific function and strictly interconnected to each other.

Locator. The Locator module was represented by a ZigBee Localization Network that was able to estimate the positions of the end-user in the domestic environment by using both a range based and area based localization procedure. The end-user's position was estimated measuring the Received Signal Strength Indicator (RSSI) from the exchanged messages between the mobile wearable nodes, placed on the user, and the fixed anchor nodes in the environment. Localization was addressed at two levels, i.e. infra-room localization and in-room localization. Firstly a simple area based localization algorithm compared RSSI values from all anchors to identify the occupied room by using a threshold algorithm [15]. Then, a range based localization method was used for a more precise "in-room" user localization. The positions of the anchor nodes in the workspace were defined according to the most probable user trajectories in the activity of daily life (e.g in front of the bed or near the household appliance) and a Hata propagation model was used for user-anchor relative distance estimation.

Navigator. The Navigator module was implemented to control the autonomous movement of robot and to acquire information from sensors installed on the 
robot, such as odometry and laser. Navigator communicated with the robot actuators and sensors by means of the Player framework [16]. Navigation was based on two algorithms: Wavefront for the global navigation, which was able to generate a sequence of waypoints that had to be reached by the robot [17], and $\mathrm{VFH}+$ for the local navigation and obstacle avoidance [18].

Sensor. The Sensor module was represented by a ZigBee Sensor Network, maintained by a PC server placed in the environment, which was able to collect information coming from environmental and wearable sensors and provide some communication actions for remote users, robot and end-user.

Graphical User Interface (GUI). The GUI module consisted of a graphical user interface including multi level submenus for easily providing functionalities to end-users, such as multimedia functionalities (news, music and video), Skype calls with relatives or caregivers and monitoring of a desired area of the home. The GUI was also used as interface during dialogues between robot and user (described in the scenarios), as drug reminder or alerts. User could navigate inside different screens (or menus) using a touch screen or by vocal commands processed by the speech module.

Speech. The Speech module represented the natural language interface between the end-user and the robot by means of appropriate tools of speech recognition and vocal synthesis. Speech recognition was based on the Simon open-source speech recognition program, produced by Simon Listens [19]. The speech synthesis was a Text-To-Speech based on MaryTTS System [20].

Inertial. The Inertial module was a data logger for inertial data (3-axis accelerometers, gyroscopes and magnetometers) coming from the (Inertial Measuring Unit (IMU) placed inside the head of the robot.

A client-server communication between modules was developed using a shared communication software bus, using the D-Bus system [21].

\subsection{The ASTRO Robot}

The ASTRO robot was built on the commercial mobile platform SCITOS G5 (Metralabs, Germany) that, based on a differential drive and a weight and payload of respectively $60 \mathrm{Kg}$ and $50 \mathrm{Kg}$, was able to move up to $1.1 \mathrm{~m} / \mathrm{s}$. ASTRO was equipped with a laser range sensor SICK S300 and a 9-axis IMU that allowed it to safely navigate in the domestic environment, avoiding obstacles and planning trajectories. The aesthetics, accessories and interfaces were appropriately designed on the basis of the end-users' requirements analysis, preliminarily identified in the first developmental phase. As a matter of fact the ASTRO (Fig. 3) was designed with a human-like shape and stylized eyes and mouth, made of a grey rigid thermoplastic ABS material and some lateral interchangeable spongy textiles on the chest and head. Based on the end-users' needs, ASTRO 


\begin{tabular}{|c|c|c|c|c|c|c|}
\hline \multicolumn{2}{|c|}{$\begin{array}{l}\text { The user needs ASTRO to } \\
\text { carry out some daily } \\
\text { activities }\end{array}$} & \multicolumn{3}{|c|}{$\begin{array}{c}\text { ASTRO autonomously } \\
\text { helps user in appropriate } \\
\text { situations }\end{array}$} & \multicolumn{2}{|c|}{$\begin{array}{c}\text { The caregiver remotely } \\
\text { drives ASTRO to assist } \\
\text { the user }\end{array}$} \\
\hline \multicolumn{7}{|c|}{ Astrologic } \\
\hline \multicolumn{7}{|c|}{ D-Bus } \\
\hline$\hat{1}$ & $\hat{1}$ & \multicolumn{2}{|c|}{ 金 } & $\widehat{1}$ & 全 & रे \\
\hline Speech & GUI & \multicolumn{2}{|c|}{ Locator } & Navigator & Inertial & Sensor \\
\hline $\begin{array}{l}\text { Robotic } \\
\text { platform }\end{array}$ & $\begin{array}{c}\text { Speakerl } \\
\text { microphone }\end{array}$ & $\begin{array}{l}\text { Touch } \\
\text { Screen }\end{array}$ & IMU & WSNs & Smartph & $\begin{array}{c}\text { Home } \\
\text { PC }\end{array}$ \\
\hline Robot & & & & & & $\underline{A m I}$ \\
\hline
\end{tabular}

Fig. 2. ASTROMOBILE architecture

included a handle, as support for standing up or walking, and a glove box for transporting small objects on the back. It was also equipped with three bumpers, implemented with two red push-buttons on the chest at the level of the handle and with a circular bumper on the base of robotic platform. In order to favor the interaction with end-users, ASTRO included an adjustable touch screen, red/blue/green-colored Light Emitted Diodes (LEDs) inside the eyes and a set of flexible microphone and speakers for the speech recognition implemented with the Simon open-source software (Simon Listens, Austria) [19].

\subsection{AmI Infrastructure}

The AmI Infrastructure was implemented by means of the two wireless sensor networks, SN and LN, suited to respectively monitor the environment and localize the users. SN and LN were designed as two different modular mesh networks respectively on the ZigBee channel 26 and 20 (Ember ZigBee-Pro stack) in order to improve the robustness and reliability of the networks. This choice was due to avoid medium access conflicts and interferences between ZigBee nodes, ensuring a better dependability in case of communication fault on a channel and an improved communication reliability by means of the mesh topology and multihop message delivery strategy. LN was composed of a ZB Coordinator (ZC), a Data Logger (DL), fixed Anchor Nodes (AN) and a wearable Mobile Node (MN) on user. ANs were equipped with HG2409P directive horizontal linear polarized antennas (Hyper Gain, USA), that were installed to spot the more frequently accessed areas of the environment with the main radiation lobe. The MN, with an omnidirectional horizontal polarized antenna, was worn by the user as a necklace. ZC was used to hold and maintain the entire LN, while the DL was used to 


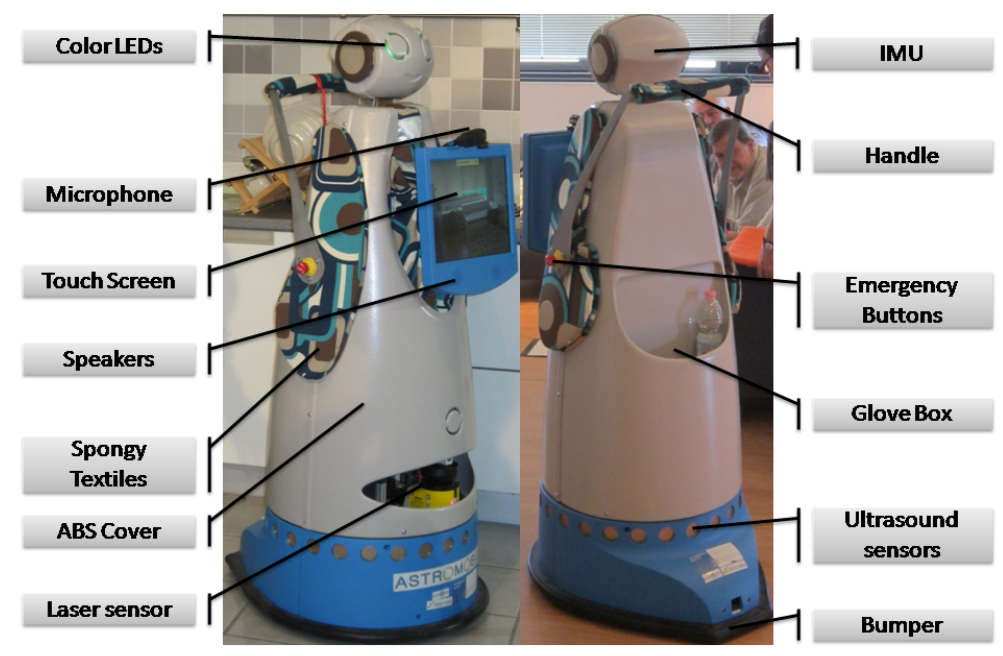

Fig. 3. Picture of ASTRO robot.

store the measured data for localization. The localization cycle $(5 \mathrm{~Hz})$ consisted of the measurement of the Received Signal Strength Indication (RSSI) from all ANs, in occasion of the reception of a message from the MN, and next in the transmission of such RSSIs to the DL, where the position of the MN was computed. SN monitored the environment by means of appropriate sensor nodes on doors, beds and sofas. Particularly, the information from these sensors were used to identify the presence of end-users on beds or sofas and then also combined with data from LN.

\section{Implementation}

The main idea of the ASTROMOBILE Experiment was far from a single, complex and autonomous robot in the environment; on the contrary the ASTRO robot was conceived to deeply cooperate with the AmI to perform complex tasks. The high-level planning layer, composed of the Astrologic module, represented the core of the AmI Infrastructure and it was implemented to coordinate and plan the execution of services, such as "agenda and reminder", "web interface control" and "communication applications" (see Table 1). For instance, considering one of the scenarios, i.e. the end-user calls ASTRO to request help in a certain situation, the Astrologic module used easy algorithms of context awareness and planning to manage the service and activate the agents to execute some consequential actions in relation to the end-users interaction (Table 2). 
Table 2. Description of the scenario in which end-user calls the ASTRO Robot to request help.

\begin{tabular}{|c|c|}
\hline Actors & Actions \\
\hline End-User & - User sits on the sofa \\
\hline ASTROMOBILE System & $\begin{array}{l}\text { - ZigBee presence sensor on sofa transmits information } \\
\text { to the Sensor module } \\
\text { - Sensor module pushes this information to the Locator } \\
\text { - Locator estimates and refreshes user position }\end{array}$ \\
\hline End-User & $\begin{array}{l}\text { - User calls ASTRO robot using the smartphone to ask } \\
\text { help in standing up from sofa }\end{array}$ \\
\hline ASTROMOBILE System & $\begin{array}{l}\text { - Vocal commands are sent from the smartphone to the } \\
\text { Speech module } \\
\text { - Speech module recognizes the command and sends it } \\
\text { to Astrologic } \\
\text { - Astrologic checks robot availability and sends the com- } \\
\text { mand Go to user to the Navigator } \\
\text { - Navigator asks to Locator the user position } \\
\text { - Navigator moves the robot to the given position and } \\
\text { informs Astrologic when the position is reached }\end{array}$ \\
\hline End-User & $\begin{array}{l}\text { - User cooperates with the system giving simple move- } \\
\text { ment voice commands (as move forward, turn right) to } \\
\text { accomplish the task as the user really needs }\end{array}$ \\
\hline ASTROMOBILE System & $\begin{array}{l}\text { - Speech module recognizes user commands and sends } \\
\text { them directly to the Navigator } \\
\text { - Navigator moves the robot following user vocal com- } \\
\text { mands }\end{array}$ \\
\hline End-User & - The user stands up from sofa using the robot handle \\
\hline
\end{tabular}


The role of Astrologic module was crucial because allowed to exploit the benefits of the integration of the AmI Infrastructure and the ASTRO robot, thus favouring the delivery of effective and high quality services. This integration expanded the sensing and planning capabilities of the ASTROMOBILE system in delivering services, allowing the ASTRO robot to know, for example, the user position even if the user was out of its sensing range. The capability of the AmI to inform the robot about the user's position or events in the environment allowed a continuous robot planning and made the ASTRO robot more effective in service delivery, avoiding long user seek procedures. Indeed the AmI Infrastructure continuously tracked the user position and sent it to ASTRO Robot, that was immediately able to know the target to reach and plan how to reach it (Fig. 4).

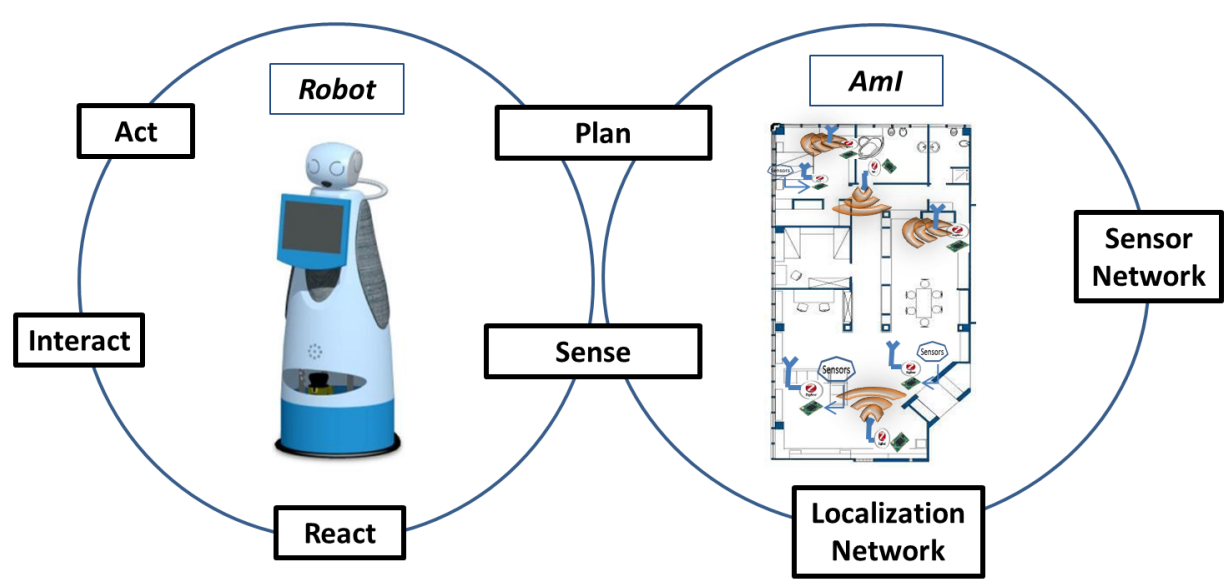

Fig. 4. Robot and AmI cooperation: AmI improves robot sensing and planning capabilities.

Table 3. Average time over 15 trials to reach the target point.

\begin{tabular}{llll}
\hline $\begin{array}{l}\text { User Position/Target ASTRO starting } \\
\text { position }\end{array}$ & Distance $[m]$ & Time $[s]$ & Notes \\
Point & middle of corridor & $\sim 4$ & $37.14 \pm 0.47$ \\
sofa in living room & $\begin{array}{l}\text { middle of corridor } \\
\text { bed in bedroom }\end{array}$ & $\sim 6$ & $75.22 \pm 10.54$ narrow passages \\
\hline
\end{tabular}




\subsection{Top-Down localization approach}

A Top-Down localization approach using sensor fusion between $L N$ and $S N$ nodes was attempted in order to localize the user in a reliable manner (Fig. 5). This localization approach consisted of two main phases: firstly the user was roughly located in the room by means of the Area Based (AB) localization; then he was more finely located using the Range Based (RB) localization.

The $\mathrm{AB}$ localization was performed measuring the RSSI from all ANs and computing the user's position as the barycentre of the area where the anchor with higher $R S S I$ was placed. As demonstrated by [15], if two adjacent ANs were placed at a distance greater than $4 m$, then the accuracy could reach $98 \%$ within 15 seconds of continuous RSSI measurement. Therefore in ASTROMOBILE each anchor was associated to an area of $16 \mathrm{~m}^{2}$. In [22], the authors demonstrated that the higher was the distance between transmitter and receiver, the higher was the probability to observe higher variance of the RSSI. Based on these results, the $L N$ anchors were cast at a distance greater than $4 m$, and RSSI was trusted to have an acceptable signal to noise ratio if MN was located within $2 m$ from an anchor. For each AN a calibration procedure was performed to identify the threshold corresponding to a value of $R S S I$ at a distance of $2 \mathrm{~m}$. If the measured RSSI from an anchor was higher than the threshold, the MN was trusted to be located in the same room of the anchor. Once the user was located in a room, the anchors in that room were used to perform a RB localization, using observed RSSI and a linear Path Loss (PL) model as suggested in [24] and in [25]. If the estimated distance using the PL model was comparable with the room dimensions, the user was located at that distance on the anchor's antenna boresight, otherwise the user position was set on the center of the room. The use of sectorial antennas allowed to improve the localization accuracy over specific areas of interest, minimizing the possibility to have comparable RSSI observations outside the anchors workspace.

Calibration. The user's localization was preliminarily anticipated by a two-levels calibration procedure. Firstly the RSSI Path Loss (PL) was modelled as a linear model using linear regression as described in equation 1 ,

$$
R S S I_{(d)}=m * d+R S S I_{(d=0)}
$$

where $R S S I_{(d)}$ was the $R S S I$ at distance $d, m$ was the slope and $R S S I_{(d=0)}$ was the $R S S I$ value at the distance $d=0$ (line/ordinate-axes interception). Slope $m$ and $d$ parameters were estimated for each anchor using MATLAB linear fitting toolbox. The second step consisted of associating a specific area to each anchor, depending on the localization accuracy to be reached and the number of installed anchors. Particularly, four areas were chosen, i.e. the kitchen, the bedroom, the corridor and the living-room. The RSSI observations collected during the calibration of the range based localization method were plotted respect to the relative distance between anchor and MN. The RSSI recorded at $2 \mathrm{~m}$ from each anchor was used as threshold and $\mathrm{MN}$ was associated to the area belonging to the anchor that observed an RSSI greater than its threshold. 


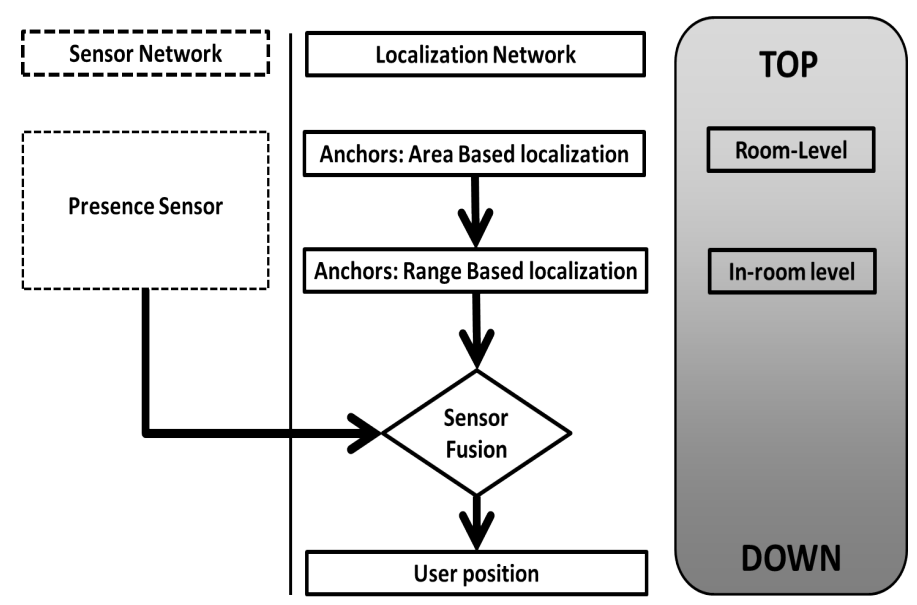

Fig. 5. Top-Down localization approach: user position was estimated in sequential steps, from a room-level location estimation, to a more sharp distance estimation between user and anchors in a room.

\section{Experiments and validation}

The ASTROMOBILE System was tested in the DomoCasa Living Lab, that reproduces a fully furnished apartment of about $120 \mathrm{~m}^{2}$ appropriately equipped with the above described ASTRO Robot and the wireless LN and SN (Fig. 6).

According to the identified users' needs, the seven scenarios, described in Table 1, were evaluated with 15 senior volunteers (male: 3 , female: 12 , mean age: 74 , min age: 66 , max age: 84 , primary school: 9 , secondary school: 5 , university: 1, living alone: 6), that accepted to participate in the experimentation after informed consensus.

During the experimentation, the LN was implemented with four anchors and a wearable mobile node, while the SN was composed of a presence sensor on bed and sofa and a door switch sensor on the main door; the ASTRO Robot was programmed to autonomously navigate in the environment, to cooperate with the AmI and to interact with users by means of an appropriate Graphical User Interface and a Speech Recognition module (Fig. 7).

An ad-hoc questionnaire was conceived in order to collect elderly volunteers' opinions. In particular, the questionnaire investigated the following aspects: functions and scenarios, aesthetics of ASTRO robot, control interfaces, interaction between user and ASTRO and general judgement about system utility and adaptability in personal habits and life style. The experiments were carried out in different sessions, in which the 15 elderly volunteers concretely tested the ASTROMOBILE system.

Concerning the functions and services provided by the ASTROMOBILE system, volunteers involved in the experimentation had a positive perception in 


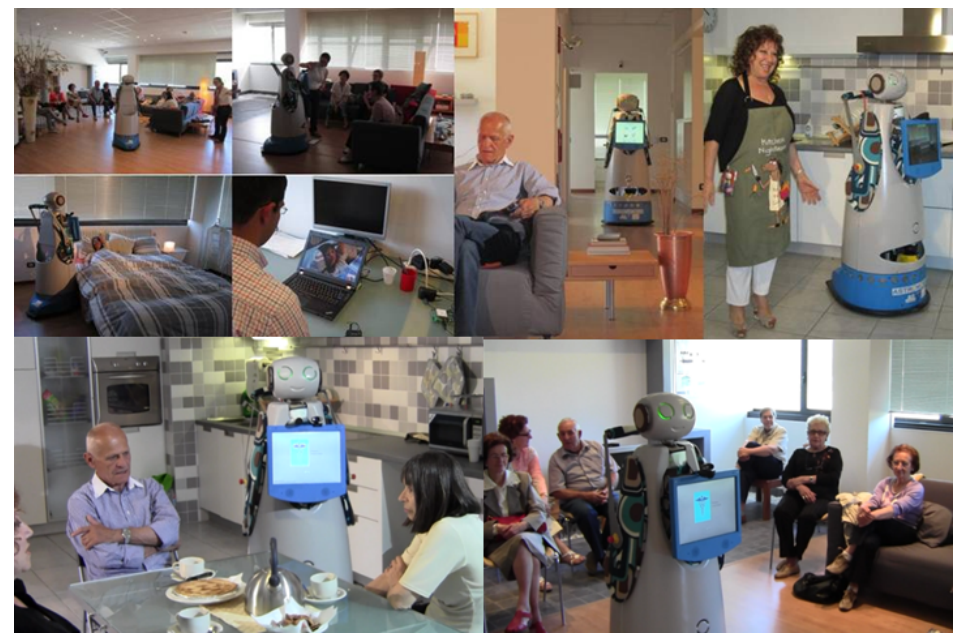

Fig. 6. Experimental sessions with elderly people in the DomoCasa Living Lab.

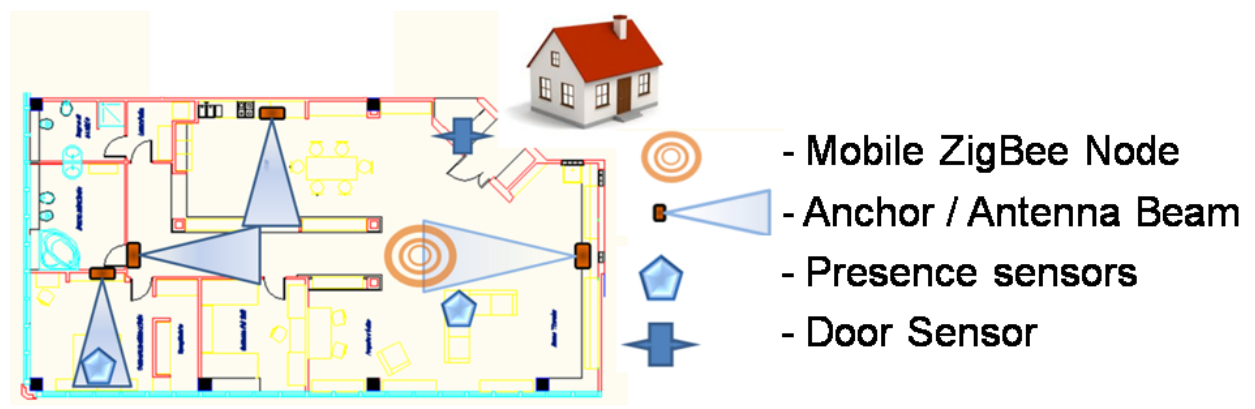

Fig. 7. Representation of the DomoCasa map, WSNs agents and position of the wireless nodes. 
terms of utility, easiness and satisfaction (Fig. 8). Most of them were considered very useful with the exception of the entertainment scenario (S4), because they believed that television and radio were already adequate for this necessity and the robot could be used in more important tasks. About the easiness of using the ASTROMOBILE system, interviewees did not find any particular difficulty, with the exception of the communication and entertainment scenarios (S3, S4), because the relative Graphical User Interface was composed of a functional menu with many options (more than four options for the list of contacts and of communication types) and therefore it was perceived not so clear and readable. Asking elderly experimenters if they were satisfied about how ASTROMOBILE system worked in the scenarios, they replied positively for most of scenarios; they only remarked the low quality of the ASTRO's speakers that were too noisy in the communication and entertainment scenarios (S3, S4).

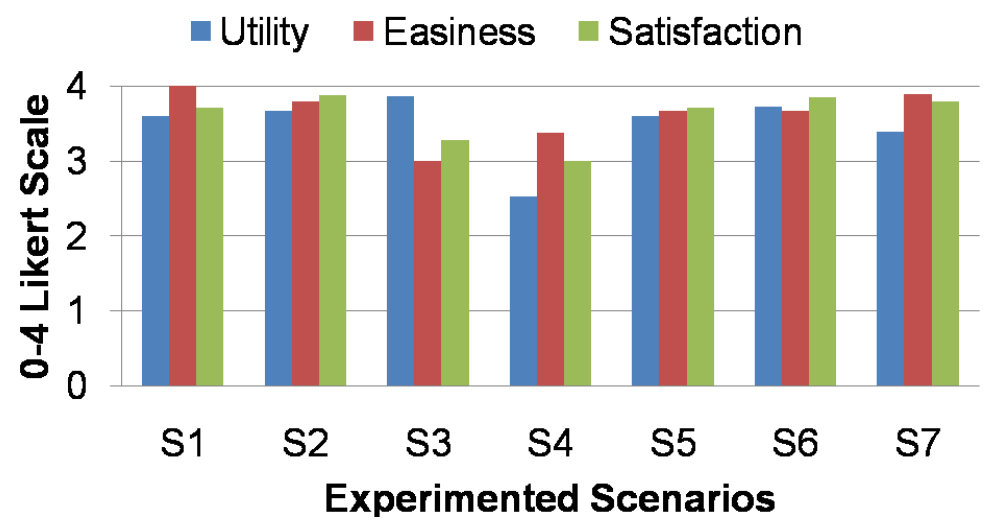

Fig. 8. Perceived Utility, Easiness and Satisfaction from volunteers, expressed in a 0-4 Likert Scale for each scenario (S1: Support in standing up, S2: Object transportation, S3: Help request /Communication, S4: Entertainment, S5: Reminder, S6: Environmental alert, S7: Remote control for caregiver).

By the aesthetic point of view, all involved users (100\%) agreed that the ASTRO Robot was nice, friendly, appeal and safe; on the other hand the $64 \%$ of them thought that ASTRO had a big size to be used in their apartments.

The three human robot interfaces, developed on the ASTRO Robot, i.e. GUI on touch screen, natural language and blue/red/green LED based eyes, were respectively evaluated in terms of usability with $3,60 / 3,20 / 3,73$ points in the 0-4 Likert scale. Particularly, the speech recognition module did not work reliably, because sometimes they had to repeat the vocal commands, and the vocal synthesis was expected to be more quick and direct toward users. However asking subjects about their favourite interface, most of them pointed out the vocal one, since this was perceived more natural (93\% of them). 
Most of elderly subjects generally thought that the ASTROMOBILE system was useful and also that it could be enough integrated in their life style. A very interesting aspect emerged from the question about a possible ASTRO purchase: only two persons (13\%) excluded completely this opportunity.

\section{Conclusions}

The work developed during the ASTROMOBILE project showed how a robotic technology for "ageing well" applications could be used in a real domestic environment with real end-users. This result could be achieved by the cooperation of the ASTRO robotic platform with smart environments and users.

The multidisciplinary approach was crucial in all developmental phases, i.e. the design phase of the system to identify the user needs, the technical development and the experimentation, according to a User Center Design approach. Cooperation with end-users became very important also during a task, where users and robot worked together to accomplish a service, in a co-working behaviour (i.e. during navigation tasks to decrease the error on the final position with respect to the goal). This was essential to perform tasks in the environment in an effective and efficient way, improving the quality of services provided to end-users. Sensors in the environment were used to monitor the state of the home and to inform the platform about current position of the user, in order to provide services where robot had to reach him/her.

Future work will focus on studying and developing the dependability of the system as well as the improvement of the whole ASTROMOBILE system. In particular, speech recognition and vocal synthesis will be investigated to enhance the capability of recognition (considering also commercial products) and to make interaction more natural, introducing instruments like dialogue manager. Future studies on AmI and WSNs will concentrate on optimization to enhance performances of networks, but also to use the information provided to improve the acceptability in terms of Human Robot Interaction.

In conclusion, this work demonstrated that the robotic technology for "ageing well" applications is nowadays promising and feasible.

\section{Acknowledgements}

The ASTROMOBILE Experiment was supported by the ECHORD project, funded by the EU-FP7-ICT-2007.2.2, (Agreement N.231143).

\section{References}

1. Plowman T, Prendergast D, Roberts S, Sherry J (2010), The Global Aging Experience Project. Ethnographic Research, Intel research.

2. European Commission - Economic Policy Committee (2012), The 2012 Ageing Report: Economic and budgetary projections for the 27 EU Member States (20102060). 
3. van der Broek G, Cavallo F, Wehrmann C (2010), AALIANCE Ambient Assisted Living Roadmap, vol. 6, IOS Press.

4. The MOBISERV Project (An Integrated Intelligent Home Environment for the Provision of Health, Nutrition and Mobility Services to the Elderly)

http://www.mobiserv.eu/index.php?lang=en

5. The Florence Project (Multi Purpose Mobile Robot for Ambient Assisted Living) http://www.florence-project.eu

6. The CompanionAble Project (Integrated Cognitive Assistive \& Domotic Companion Robotic Systems for Ability \& Security)

http://www.companionable.net

7. The KSERA Project (Knowledgeable SErvice Robots for Aging) http://ksera.ieis.tue.nl

8. The SRS Project(Shadow Robotic System for Independent Living) http://srs-project.eu

9. Care-O-Bot 3

http://www.care-o-bot.de/english/index.php

10. Robot Maid http://www.lunegate.com

11. European Robotics Technology Platform (EUROP)(2009), Robotic Visions To 2020 And Beyond - The Strategic Research Agenda For Robotics In Europe.

12. Volkhardt, M., Mueller, S., Schroeter, C., \& Gross, H. M. (2011, October). Playing hide and seek with a mobile companion robot. In Humanoid Robots (Humanoids), 2011 11th IEEE-RAS International Conference on (pp. 40-46). IEEE.

13. Yachir, A., Tari, K., Amirat, Y., Chibani, A., \& Badache, N. (2009, October). QoS based framework for ubiquitous robotic services composition. In Intelligent Robots and Systems, 2009. IROS 2009. IEEE/RSJ International Conference on (pp. 20192026). IEEE.

14. Heerink, M., Krse, B., Evers, V., Wielinga B. (2009). Measuring acceptance of an assistive social robot: a suggested toolkit. The 18th IEEE International Symposium on Robot and Human Interactive Communication, 2009 (pag. 528-533). ROMAN 2009.

15. Yan, R. H., Yu, C. H., Ding, I. J., \& Tsai, K. C. (2006). Wireless Sensor network Based Smart Community Security Service. In The 2nd Workshop on Wireless, Ad Hoc, and Sensor Networks (p. 13).

16. Player web page, http://playerstage.sourceforge.net/

17. LaValle S M (2006), Planning algorithms, Cambridge University Press, Chambridge, UK.

18. Ulrich I, Borenstein J (1998), VFH+: reliable obstacle avoidance for fast mobile robots, Proc.1998 IEEE International Conference on Robotics and Automation, ICRA98, Leuven, Belgium, pp.1572-1577.

19. Simon Listens official web page http://simon-listens.org

20. Modular Architecture for Research on speech sYnthesis (MARY) official web page http://mary.dfki.de

21. D-Bus web page, http://www.freedesktop.org/wiki/Software/dbus

22. Graefenstein, J., \& Bouzouraa, M. E. (2008, May). Robust method for outdoor localization of a mobile robot using received signal strength in low power wireless networks. In Robotics and Automation, 2008. ICRA 2008. IEEE International Conference on (pp. 33-38). IEEE. 
23. Dabin, J. A., Ni, N., Haimovich, A. M., Niver, E., \& Grebel, H. (2003, November). The effects of antenna directivity on path loss and multipath propagation in UWB indoor wireless channels. In Ultra Wideband Systems and Technologies, 2003 IEEE Conference on (pp. 305-309). IEEE.

24. Whitehouse, K., Karlof, C., \& Culler, D. (2007). A practical evaluation of radio signal strength for ranging-based localization. In ACM SIGMOBILE Mobile Computing and Communications Review, Volume 11, Issue 1, Pag. 41-52.

25. Ding, X., Zhao, H., Zhu, J., Zhang, K., \& Li, D. (2011, September). A Novel Localization Algorithm Based on RSSI for Wireless Sensor Networks. In Wireless Communications, Networking and Mobile Computing (WiCOM), 2011 7th International Conference on (pp. 1-4). IEEE.

26. Arndt, M., \& Berns, K. (2012). Mobile Robots in Smart Environments: The Current Situation. Autonomous Mobile Systems 2012 (pp 39-47). Springer. 